\title{
Quantitative evaluation of the FTA-ABS-IgM and VDRL test in treated and untreated syphilis
}

\author{
A. LUGER, B. SCHMIDT, AND I. SPENDLINGWIMMER \\ From the Ludwig-Boltzmann-Institut für dermato-venerologische Serodiagnostik im Krankenhaus der Stadt \\ Wien-Lainz
}

SUMMARY Observations made on fluorescent treponemal antibody absorption (FTA-ABS) immunoglobulin $\mathbf{M}$ (IgM) titres in patients with untreated early syphilis showed non-reactive or weakly reactive results in patients with primary (one of five cases) and secondary (two of 16 cases) lesions. In patients with primary $(19.5 \%)$ and secondary $(15 \%)$ syphilis sera remained reactive with increased titres for more than one year after treatment. The respective figures in the results of the Venereal Disease Research Laboratory (VDRL) test were zero in primary and $20 \%$ in secondary syphilis. The non-reactive FTA-ABS IgM results may possibly be explained by competitive inhibition of IgM by immunoglobulin $\mathbf{G}(\mathrm{IgG})$. The persistence of reactivity in a comparable percentage has been observed by other investigators (Grin et al., 1974; Wilkinson and Rodin, 1976). The current results therefore suggest that FTA-ABS IgM titres are less reliable for assessing the effect of treatment than the course of the VDRL titres. The phenomenon of a decrease in FTA-ABS IgM titres soon after treatment with a later rise before final non-reactivity is a matter for further investigation.

\section{Introduction}

The detection of immunoglobulin (IgM) antibodies against Treponema pallidum has for some time been considered of value in diagnosing syphilis and assessing the effect of treatment (Bowszyc, 1975; Manikowska-Lesinska et al., 1976; Wilkinson, 1976; Wilkinson and Rodin, 1976; Müller, 1977a, b). Experience suggests that IgM antibodies appear before other immunoglobulins and are generally present in the sera of untreated patients with early syphilis, but can be detected in only $20 \%$ of people with untreated late and latent syphilis (Wilkinson and Rodin, 1976). Investigations to confirm this have been made at this institute and have given similar results.

\section{Material and methods}

An increasing number of sera (at present more than 100000 samples a year) are examined at the institute using the automated microhaemagglutination assay

Address for reprints: Ludwig-Boltzmann-Institut für dermatovenerologische Serodiagnostik im Krankenhaus der Stadt Wien-Lainz, Wolkersbergenstr. 1, A-1130 Wien, Austria

Received for publication 18 May 1977 with $T$. pallidum antigen (AMHA-TP) and the Venereal Disease Research Laboratory (VDRL) test. All sera reactive to one of these two tests are further evaluated by the fluorescent treponemal antibody absorption (FTA-ABS) test which is carried out in accordance with international recommendations (United States Public Health Service, 1969).

The microscope used for the test was a ReichertZetopan. Fluorescence was induced by a mercury high pressure lamp HBO $200 \mathrm{~W}$, with an excitor filter UG 1 and a barrier filter GG 13 with a Wratten folia 2 B.

The detection of IgM antibodies followed the same principle but a specific monovalent anti-humanIgM labelled with sodium-fluorescein-isothiocyanate was used instead of the polyvalent anti-human globulin. The quantitative FTA-ABS IgM was determined by diluting the samples in a geometrical progression by the factor 3 starting with $1: 5$, $1: 45$, etc.

The conjugates were products of Wellcome Laboratories and contained pure sheep anti-humanIgM. A check of the reliability of the reagent with sera of known IgM reactivity was performed before any investigation started.

The VDRL test and the quantitative evaluation of 
reactive results in this assay were performed in accordance with international recommendations (United States Public Health Service, 1969).

All patients in the study were treated with benzathine penicillin 4.8 megaunits intramuscularly in a single dose.

\section{Results}

PRIMARY SYPHILIS

Figure 1 shows the results of an examination of 75 sera from 39 patients who were diagnosed and treated during the primary stage of syphilis. The diagnosis was confirmed by darkfield examination in each case.

Five sera were tested before treatment. With the FTA-ABS IgM test one of these was weakly reactive, the others were strongly reactive with titres ranging from $1: 5$ to $1: 3645$.

Seventy samples were received from 39 patients at different times after treatment with penicillin. The FTA-ABS IgM titres were non-reactive or weakly reactive in 54 of the 70 examinations $(77 \cdot 1 \%)$, while 16 proved to be reactive. Eight of 41 specimens $(19.5 \%)$ were still reactive more than one year after the end of treatment. The reactivity of one of these samples was present at a dilution of $1: 45$ during the second year after treatment and two more had a titre of $1: 15$ in the third and fifth years. The sera of two patients were reactive in a dilution of $1: 5$ 30 and 31 years after treatment. However, these two patients might not have originally received antibiotic treatment.

The VDRL titre was weakly reactive in two of five sera before treatment and three others showed titres of $1: 2$ and $1: 4$. Two of 54 persons were weakly reactive after the third month following treatment $(3.7 \%)$, all others were non-reactive. Weakly reactive results are generally considered to fall within the margin of error of the method in both the FTA-ABS IgM and the VDRL tests.

\section{SECONDARY SYPHILIS}

One hundred and forty-two sera of 48 patients who suffered from secondary lesions were tested by the same procedure. The results are presented in Fig. 2.

Sixteen samples were examined before treatment, one was non-reactive and one was weakly reactive

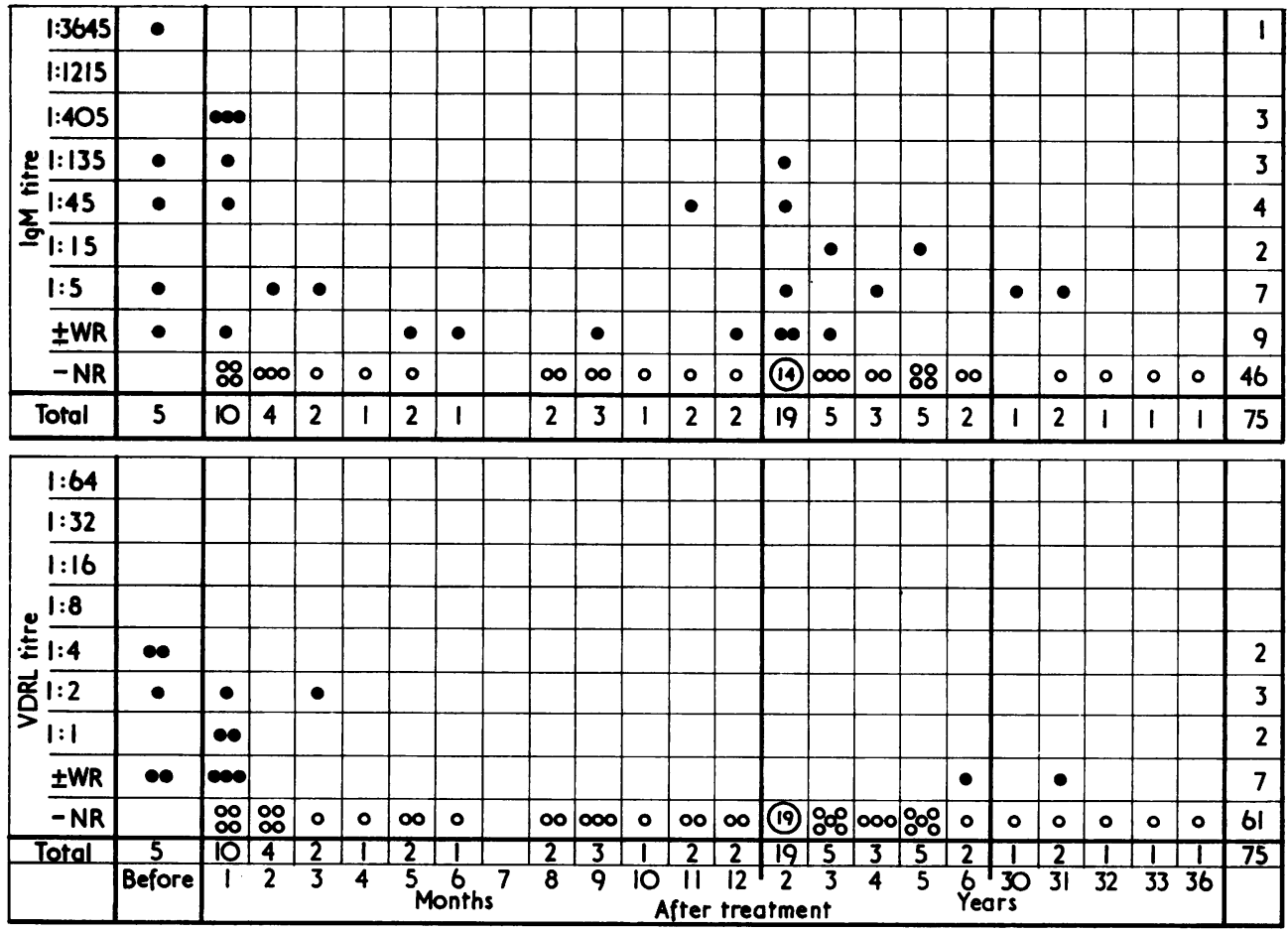

$\mathrm{N}$ (Persons) $=39 \mathrm{~N}$ (Examinations) $=75$

Fig. 1 IgM and VDRL in primary syphilis before and after treatment. 

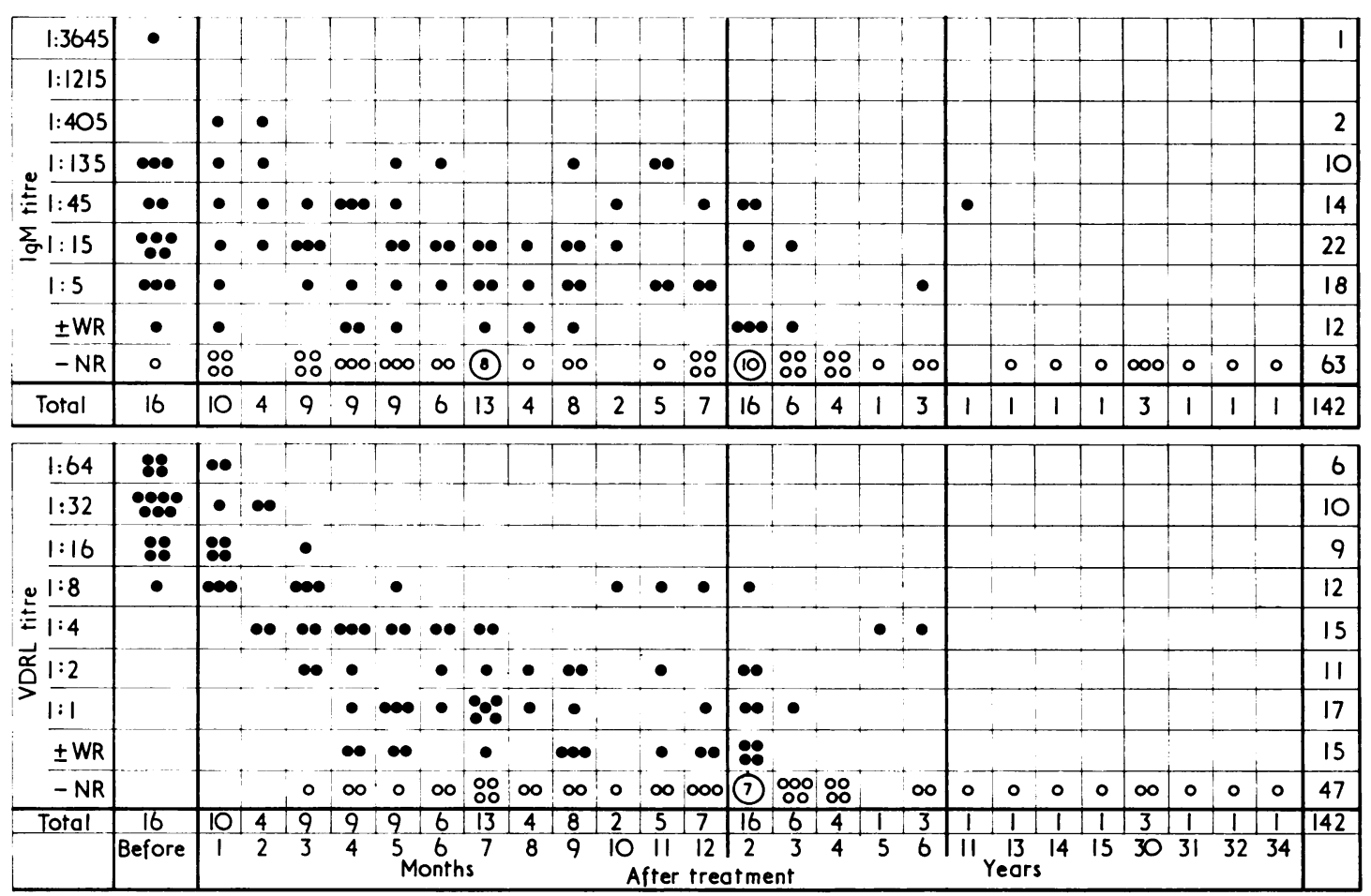

$\mathrm{N}$ (Persons) $=48 \mathrm{~N}($ Examinations $)=142$

Fig. 2 IgM and VDRL in secondary syphilis before and after treatment.

to the FTA-ABS IgM test; the others showed titres between $1: 5$ and $1: 3645$ (mean value $1: 282$ ).

One hundred and twenty-six sera were from patients who had received treatment between one month and 34 years before the test. Fifty-three $(42.1 \%)$ of these sera were reactive up to dilutions of between $1: 5$ and $1: 405$. The results of the remaining 73 were non-reactive (62) or weakly reactive (11).

Six of $40(15 \%)$ samples were reactive more than one year after treatment at dilutions of $1: 5$ (1), $1: 15$ (2), and $1: 45(3)$.

The VDRL test was performed on 16 sera before treatment, all were reactive at titres between $1: 8$ and 1:64 (mean value $1: 34 \cdot 5$ ). Sixty-two of the samples which had been examined after treatment were non-reactive (47) or weakly reactive (15). Sixty-four sera $(51.6 \%)$ were reactive at titres between $1: 1$ and $1: 64$.

The reactivity persisted for more than one year after treatment in eight of $40(20 \%)$ specimens, the respective dilutions reached from $1: 1$ (3), $1: 2$ (2), $1: 4(2)$, to $1: 8(1)$.
LATENT SYPHILIS

We were unable to distinguish between samples from patients with early latent and late latent syphilis, therefore a precise evaluation cannot be given. In Fig. 3 the FTA-ABS IgM and VDRL titres are compared. The agreement between non-reactive and weakly reactive samples using either method was 61 out of $121(50.41 \%)$, and 10 further sera $(8 \cdot 26 \%)$ showed good agreement because the outcome of the tests did not deviate from the middle for more than one step. The other 50 results $(41 \cdot 32 \%)$ were scattered (Fig. 3).

Altogether 37 samples $(30.6 \%)$ were reactive, 84 were non-reactive (67) or weakly reactive (17).

FOLLOW-UP

Nine patients with secondary syphilis were followedup. FTA-ABS IgM and VDRL titres showed only partial agreement during the course of observation. The results are shown in Fig. 4. The FTA-ABS IgM titre usually reverted to non-reactivity later than the 
VDRL dilutions. The VDRL titre therefore seems to be better for measuring the effect of treatment.

\begin{tabular}{|c|c|c|c|c|c|c|c|c|c|c|c|}
\hline \multirow{2}{*}{\multicolumn{2}{|c|}{-NR }} & \multirow{2}{*}{$\frac{-\mathrm{NR}}{40}$} & \multicolumn{8}{|c|}{ 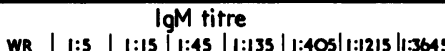 } & \multirow{2}{*}{\begin{tabular}{|l|} 
Total \\
69
\end{tabular}} \\
\hline & & & 10 & 9 & 7 & 1 & 2 & & & & \\
\hline & $\pm W R$ & 9 & 2 & & 2 & & & & & & 13 \\
\hline & 1:1 & 6 & 2 & 3 & & & & & & & II \\
\hline 墨 & $1: 2$ & 4 & 1 & 3 & & & & 1 & & & 9 \\
\hline$\vec{\alpha}$ & 1:4 & 4 & 2 & & 1 & & & 1 & & & 8 \\
\hline & 1:8 & 1 & & & 2 & 1 & & & & & 4 \\
\hline & $1: 16$ & 2 & & 1 & & & & & & & 3 \\
\hline & 1:32 & 1 & & & & & & & & & 1 \\
\hline & $1: 64$ & & & 1 & 1 & & & 1 & & & 3 \\
\hline & & 67 & 17 & 17 & 13 & 2 & 2 & 3 & - & - & 121 \\
\hline
\end{tabular}

Fig. 3 IgM and VDRL titres in latent syphilis.

IgM AND VDRL TITRES SHORTLY AFTER SINGLE DOSE TREATMENT

Case 1

A 25-year-old man presented with a chancre 1:5 $\mathrm{cm}$ across in the right inguinal region. Darkfield examination showed $T$. pallidum. A mild Herxheimer reaction began three hours after the injection of 4.8 megaunits benzathine-penicillin with a rise of the axillary temperature to $37 \cdot 3^{\circ} \mathrm{C}$ which lasted for

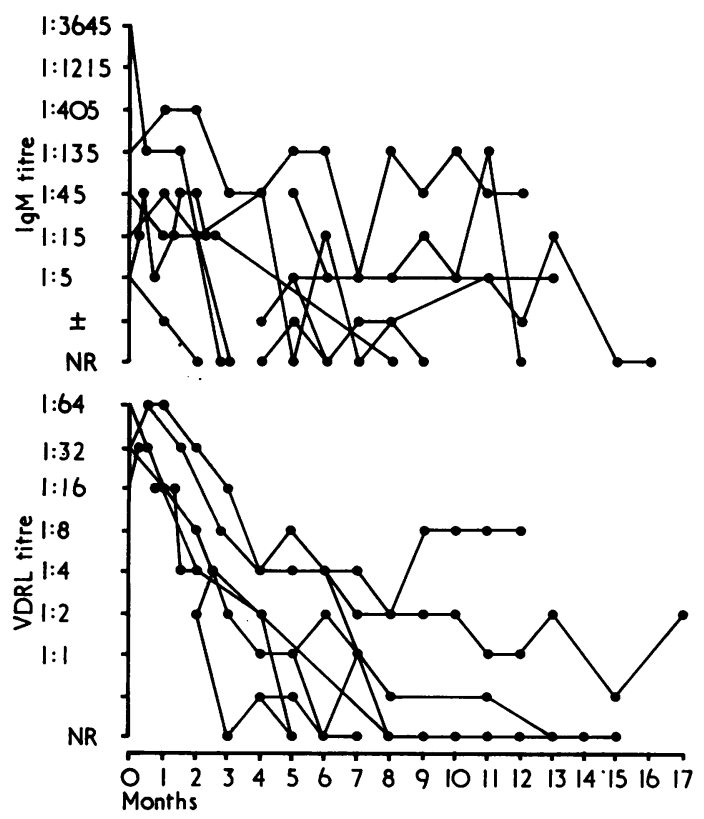

Fig. 4 Succession of IgM and VDRL titres in the sera of nine patients with secondary syphilis.
12 hours, but with no other general or local symptoms. Fig. 5 shows that the IgM titre decreased to zero within three weeks after treatment, but that it later rose to $1: 5$ and then finally returned to nonreactive or weakly reactive results one month later. The VDRL titre was weakly reactive, apart from 48 hours after the injection of penicillin when it was reactive in neat serum.

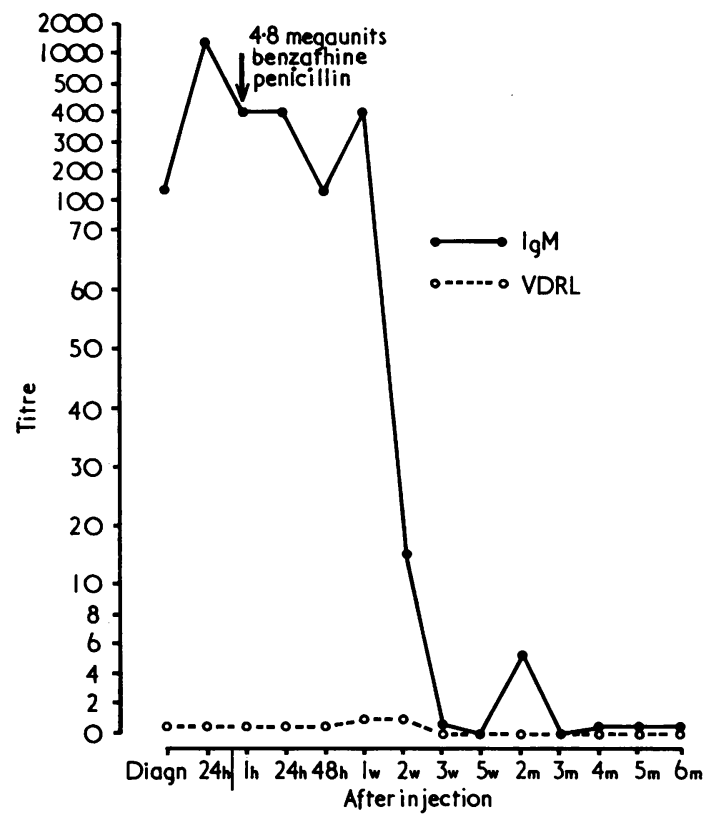

Fig. 5 IgM and VDRL titres in the serum of a patient with primary syphilis shortly after penicillin administration.

Case 2

A 77-year-old man presented with a macular rash caused by secondary syphilis. The patient was observed two days before and $\mathbf{1 1}$ days after a single injection of 4.8 megaunits of benzathine penicillin (Fig. 6).

A mild Herxheimer reaction began four hours after the injection; the rash became deeper in colour and the axillary temperature rose to $37 \cdot 7^{\circ} \mathrm{C}$ where it remained for 12 hours. The FTA-ABS IgM titre increased at first irregularly from 1:135 up to $1: 1 \cdot 215$ and was then checked at three-hourly intervals after injection of penicillin. The titre decreased steadily until it returned to non-reactivity two days after treatment. However, during the next few days an increase in the FTA-ABS IgM concentration in the serum was observed until the sixth 
day when the titre reached a peak of $1: 135$, after which it dropped to weakly reactive within two days.

The VDRL titre hovered around 1:32 but remained within the margin of error of one dilution until the fifth day after treatment and then declined steadily to $1: 8$.

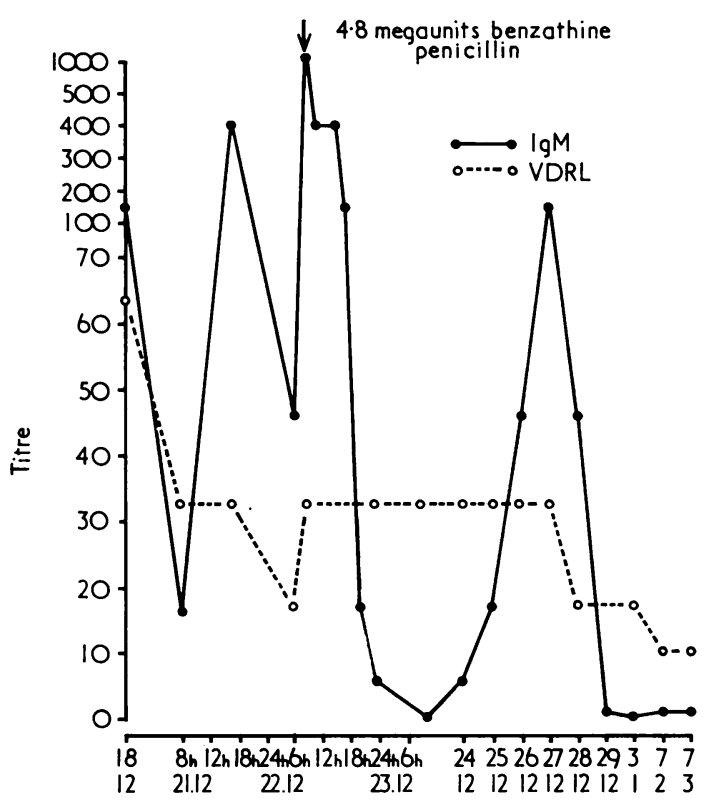

Fig. 6 IgM and VDRL titres in the serum of a patient uith secondary syphilis shortly after application of 4.8 megaunits of benzathine penicillin.

Both results suggest that the antisyphilitic treatment might influence the FTA-ABS IgM titre by a marked decrease after treatment and a later increase up to a second peak. This phenomenon is a matter for further study but it has proved difficult to find patients who were willing to stay in hospital for long enough to gather data.

\section{Discussion}

On long-term observations the FTA-ABS IgM titres showed neither a prompt nor a steady decrease after treatment but remained reactive beyond the first year after treatment at dilutions of $1: 5$ and more in $19.5 \%$ of cases of primary syphilis, and $15.0 \%$ of cases of secondary syphilis. Similar results have been reported by others (Grin et al., 1974 ; Wilkinson, 1976; Wilkinson and Rodin, 1976).
Possible explanations for non-reactive or weakly reactive results in primary and secondary syphilis before treatment could be a competitive inhibition of the specific IgM production or a blocking of the IgM receptors on the surface of the antigen by IgG as explained by Wilkinson (1976) and Müller (1977b).

Contamination of the FTA-ABS IgM reagent with IgG could be a source of error. We are currently trying to establish if this can influence the FTA-ABS IgM results and if so to what extent. The work has not yet finished.

A possible contamination of the conjugate with IgG could have a marked effect on all investigations since IgG induces a more intensive fluorescence than IgM. Nevertheless, the present standard of controls suggests that contamination would only affect the result by one dilution.

The influence of the sorbent on the outcome of the FTA-ABS IgM results has already been stressed (Vozely, 1974; Wilkinson and Johnston, 1975). Müller (1976) demonstrated that only one out of 10 different sorbent preparations produced satisfactory results, all others did not completely absorb nonspecific group antigens.

\section{References}

Bowszyc, J. (1975). The FTA-ABS-IgM test in untreated and treated acquired syphilis. Przeglad Dermatolgiczny, 62, 347-352.

Grin, I., Nadaždin, M., and Pasic, A. (1974). Investigations of immunoglobulins by FTA (IgG, IgM) and quantitative immunodiffusion (IgG, IgM, IgA) in endemic syphilis twenty years after treatment. Acta dermatologica et venerologica Jugoslavica, 1, 5-11.

Manikowska-Lesinska, W., Linda, B., Szymska, K., and Zajac, W. (1976). An appraisal of repeatability and sensitivity of immunofluorescence tests in Polish laboratories of serology of syphilis. Przeglad Dermatolgiczny, 63, 143-150.

Müller, F. (1976). Vergleichende Untersuchungen über die Wirksamkeit von Sorbent mit Treponema-Ultrasonikat auf die SyphilisSpezifitäten im FTA-ABS-Test. Ärtliche Laboratorium, 22, 351-354.

Müller, F. (1977a). Erregerspezifisches monomeres IgM (8S-IgM) bei Patienten mit Treponema-Infektion (Syphilis). In Vortrag anlasslich des 3 Herbstmeetings der Österreichischen Gesellschaft für Allergologie und Immunologie, Wien, 1976. In press.

Müller, F. (1977b). Serodiagnostik der Syphilis aus der Sicht des Immunologen. Hautarzt 28, 167-172.

United States Public Health Service (1969). Manual of Tests for Syphilis. US Department of Health, Education and Welfare: Atlanta.

Vozely, M. (1974). Elimination of false positive reactions in the FTA test for syphilis. Acta dermatologica et venerologica Jugoslavica, 1, 59-64.

Wilkinson, A. E. (1976). Some aspects of research on syphilis: Serological evidence of activity of the disease. In Sexually Transmitted Diseases, pp. 214-218. Edited by R. D. Catterall and C. S. Nicol. Academic Press: London.

Wilkinson, A. E., and Johnston, N. A. (1975). A comparison between sorbent and Reiter-sonicate in the absorbed fluorescent treponemal antibody test. Annals of the New York Academy of Sciences, 254, 395-399.

Wilkinson, A. E., and Rodin, P. (1976). IgM-FTA test in syphilis in adults: Its relation to clinical findings. British Journal of Venereal Diseases, 52, 219-223. 